\title{
Designing digital filter banks using wavelets
}

\author{
Sergio R. M. Penedo, Marcio Lobo Netto and João F. Justo* (i)
}

\begin{abstract}
In digital filters theory, filtering techniques generally deal with pole-zero structures. In this context, filtering schemes, such as infinite impulse response (IIR) filters, are described by linear differential equations or linear transformations, in which the impulse response of each filter provides its complete characterization, under filter design specifications. On the other hand, finite impulse response (FIR) digital filters are more flexible than the analog ones, yielding higher quality factors. Since many approaches to the circuit synthesis using the wavelet transform have been recently proposed, here we present a digital filter design algorithm, based on signal wavelet decomposition, which explores the energy partitioning among frequency sub-bands. Exploring such motivation, the method involves the design of a perfect reconstruction wavelet filter bank, of a suitable choice of roots in the Z-plane, through a spectral factorization, exploring the orthogonality and localization property of the wavelet functions. This approach resulted in an energy partitioning across scales of the wavelet transform that enabled a superior filtering performance, in terms of its behavior on the pass and stop bands. This algorithm presented superior results when compared to windowed FIR digital filter design, in terms of the intended behavior in its transition band. Simulations of the filter impulse response for the proposed method are presented, displaying the good behavior of the method with respect to the transition bandwidth of the involved filters.
\end{abstract}

Keywords: Filtering, Wavelet analysis, Circuit synthesis

\section{Introduction}

Filter design has been extensively explored in circuit synthesis and signal processing, as a part of circuit theory [1-7]. Within this context, one of the major challenges of the IIR filters is determining its respective coefficients: in a digital form, the IIR filters can be designed from their analog versions, a procedure that is not easily performed $[2,4]$. On the other hand, the FIR filters are more powerful than the IIR ones [4], but they also require more processing power. In this scenario of drawbacks, the frequency partitioning into sub-bands, obtained by the wavelet transform, could be useful: in the wavelet decomposition of a given signal, frequency sub-bands are obtained with peculiar amplitude values, which could be explored in selective filtering techniques.

The wavelet filter banks provide the advantage of separating the signal under consideration into two or more signals, in the frequency domain. Since signals can show

\footnotetext{
* Correspondence: jjusto@lme.usp.br

Departamento de Engenharia de Sistemas Eletrônicos, Escola Politécnica, Universidade de São Paulo, São Paulo, SP CEP 05508-970, Brazil
}

different amplitude levels in both time and wavelet transform domains, it is interesting to partition the energy into several frequency sub-bands for several applications. This could be achieved using low-pass or highpass filters, associated with wavelets respectively with few or many vanishing moments [8-10]. This apparent duality could be better described using spectral factorization [9-12], which allows separating polynomial roots into two corresponding sequences of low-pass filters in a wavelet filter bank, according to a criterion of perfect reconstruction [12]. The selective filtering method proposed here designs a wavelet family that decomposes the signal to be filtered into sub-bands fitted in amplitude, obtaining good quantitative results in terms of accuracy of filtering and simultaneously with a low computational cost. Although this technique has been studied in several digital signal processing applications, so far it has not been widely explored in digital circuit theory [13-15], which is the main focus of this investigation, primarily the digital filter synthesis, 
showing the good choice for wavelets in digital filtering applications.

This manuscript is organized as follows. Section 2 presents the theory of wavelet filter banks, designed to obtain perfect reconstruction. Section 3 highlights the role of the multiplicity of a root at $z=-1$ in digital filter design. Section 4 presents the methodology for designing a specific wavelet family based on the spectral factorization algorithm $[8,12]$. Section 5 presents the frequency responses for the designed filter, when compared to first-order filtering stages, generally implemented with circuit elements. Finally, section VI discusses the results and possible outcomes of this modeling.

\section{Methods: wavelet theory and filter banks}

In signal processing, a filter bank is an array of bandpass filters that separates the input signal into multiple components, each one carrying a single frequency subband of the original signal. For example, an application of a filter bank lies in designing a graphic equalizer, which can attenuate the components differently and recombine them into a modified version of the original signal $[16,17]$. The decomposition process, performed by the filter bank, is labeled analysis (meaning analysis of the signal in terms of its components in each subband); the output of the analysis is referred to as a subband signal, where each frequency sub-band is related to a specific filter in a bank. The reconstruction process is labeled synthesis, meaning the reconstitution of a complete signal resulting from the filtering process [18]. Particularly in digital signal processing, the term filter bank is also commonly applied to a bank of receivers $[19,20]$. The difference is that receivers also down-convert the sub-bands to a low-center frequency that can be resampled at a reduced rate. The same procedure could be sometimes performed by undersampling the bandpass sub-bands.

The analysis of signals by filter banks requires minimally filtering techniques using Fourier analysis [18], which use complex sinusoids as basis functions. However, a difficulty that has often been raised with this approach is that, because of the infinite extent of the basis functions, any time-local information is spread out over the whole frequency axis [18]. Under such constraints, the wavelet basis is a set of functions that can represent signals with good resolution in both time and frequency domains. The wavelet transform is well defined within the multiresolution framework, which allows signal analysis in several scales. Wavelets are characterized by time locality, allowing an efficient capture of transient behavior in a signal. Furthermore, the time-frequency resolution trade-off, provided by the multiresolution analysis, enables a better signal representation over the
Fourier analysis, since it reveals signal aspects that Fourier signal representation usually neglects, such as trends, breakdown points, and discontinuities. In this context, a wavelet filter bank is an array of wavelet filters used to decompose a signal into sub-bands over different regions of the frequency spectrum, without losing the time domain characterization as performed by the Fourier transform, which is useful in circuit applications.

Particularly, wavelets are function sets obtained from a prototype function (labeled mother wavelet) through dilations and translations. The general form of a wavelet family is given by

$$
\psi_{j, k}(x)=\psi\left(2^{j} x-k\right) \quad, j, k \in Z
$$

where $\psi_{j, k}(x)$ is the 'mother wavelet', while $j$ and $k$ are respectively the scale and translation factors.

The algebra behind the wavelet filter bank design has been extensively discussed elsewhere $[8,9]$. A two-channel wavelet filter bank structure involves four filters: the analysis stage has a low-pass $H_{0}(z)$ and a high-pass $H_{1}(z)$ filter (with $h_{0}[n]$ and $h_{1}[n]$ impulse responses, respectively), while the synthesis stage is formed by low-pass $G_{0}(z)$ and high-pass $G_{1}(z)$ filters (with $g_{0}[n]$ and $g_{1}[n]$ impulse responses, respectively), as shown in Fig. 1. The output signals of those filters, $y_{0}[n]$ and $y_{1}[n]$, must be decimated by a factor of two, retaining only the even samples, since each filter output contains half of the frequency content, but an equal amount of samples as the input signal. The combination of the two outputs contains the same frequency content as the input signal, but the amount of data is doubled. Therefore, the downsampling procedure, denoted by the operator $\downarrow 2$, is applied on filter outputs in the analysis bank:

$$
y_{0}[n]=h_{0}[n] * x[n], v_{0}[n]=y_{0}[2 n]
$$

and

$$
y_{1}[n]=h_{1}[n] * x[n], v_{1}[n]=y_{1}[2 n]
$$

where the ' $*$ ' symbol is the convolution operator.

The input signal $x[n]$ with a fixed length $q$ (for analysis purposes) produces two signals, $v_{0}[n]$ and $v_{1}[n]$, with length $q / 2$. This analysis step is opposite to the synthesis one: $v_{0}[n]$ and $v_{1}[n]$ are interpolated (by a factor of 2 ) with zeros in odd samples (denoted by the operator $\uparrow 2$ ). The results are filtered by $G_{0}(z)$ and $G_{1}(z)$, and their sum gives $\tilde{x}[n]$ :

$$
\tilde{x}[n]=g_{0}[n] * \tilde{v}_{0}[n]+g_{1}[n] * \tilde{v}_{1}[n],
$$

where 


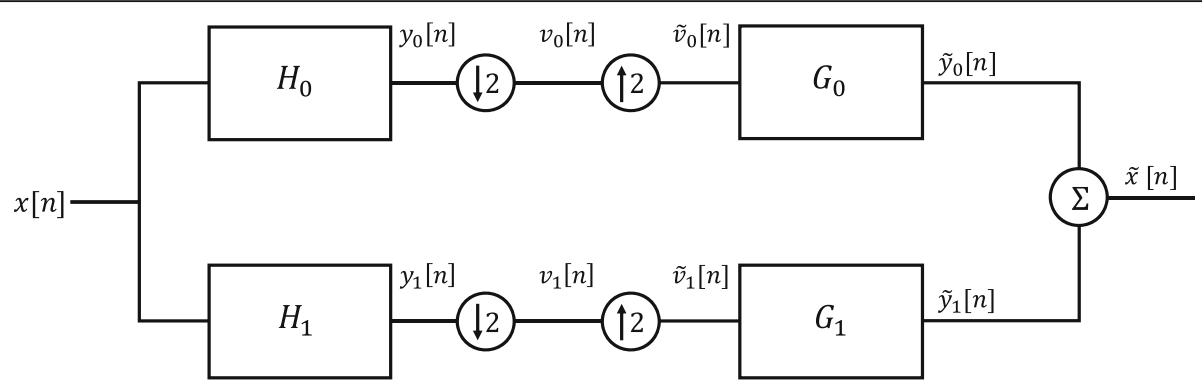

Fig. 1 A two-channel filter bank with analysis and synthesis stages

$$
\tilde{v}_{i}[m]= \begin{cases}v_{i}[m / 2] & , m \text { even } \\ 0 & , m \text { odd }\end{cases}
$$

If $\tilde{x}[n]=x[n-l]$ (i.e., if the input signal is delayed by $l$ integer samples), the filter bank allows perfect reconstruction [8, 21, 22]. The conditions for this case are now explained through signal analysis along the filter bank, in the Z-transform domain. An analysis of Eqs. (4) and (5) in the $Z$-domain leads to

$$
\begin{aligned}
\tilde{X}(z) & =\frac{1}{2} G_{0}(z)\left\{H_{0}(z) X(z)+H_{0}(-z) X(-z)\right\} \\
& +\frac{1}{2} G_{1}(z)\left\{H_{1}(z) X(z)+H_{1}(-z) X(-z)\right\}=z^{-l} X(z) .
\end{aligned}
$$

To avoid output aliasing, the following conditions for perfect reconstruction must be defined $[11,21]$ :

$$
G_{0}(z) H_{0}(z)+G_{1}(z) H_{1}(z)=2 z^{-l}
$$

and

$$
G_{0}(z) H_{0}(-z)+G_{1}(z) H_{1}(-z)=0
$$

The anti-aliasing condition from Eq. (8) leads to "alternating signal" constructions [9], corresponding, in polynomial terms, to $H_{1}(z)=G_{0}(-z)$ and $G_{1}(z)=-H_{0}(-z)$. Therefore, Eq. (8) is satisfied, and Eq. (7) reduces to an equation of the product filter $P_{0}(z)=G_{0}(z) H_{0}(z)$, such that

$$
P_{0}(z)-P_{0}(-z)=2 z^{-l}
$$

This is the key equation to design filters with perfect reconstruction [9], in which the left term is an odd function, so $l$ must be odd. This means that the only odd term in $P_{0}(z)$ is equal to $z^{-l}$. The solution of Eq. (9) leads to the relationships

$$
\begin{aligned}
& h_{0}\left[L_{0}-1-k\right]=(-1)^{k} g_{1}[k] \\
& h_{1}\left[L_{1}-1-k\right]=(-1)^{k} g_{0}[k]
\end{aligned}
$$

where $L_{0}$ and $L_{1}$ are the lengths of the filters $h_{0}[n]$ and $h_{i}[n]$, respectively. This leads to

$$
\begin{aligned}
& \tilde{y}_{0}[k]=\sum_{n} x[n] g_{0}[-n+2 k] \\
& \tilde{y}_{1}[k]=\sum_{n} x[n] h_{1}[-n+2 k] \\
& \tilde{x}[n]=\sum_{k}\left\{y_{0}[k] g_{0}[-n+2 k]+y_{1}[k] h_{1}[-n+2 k]\right\}
\end{aligned}
$$

This formulation motivates the study of digital filter design from a perspective of filter root analysis, which is explored in the following sections.

\section{Root analysis in digital filters}

Digital filters have been widely used in signal processing and communication systems, in applications such as channel equalization, noise reduction, radar, audio and video processing, biomedical signal processing, and analysis of economic data [2, 3, 23-25]. In those examples, the roots of the digital filters play a major role in their design. Their location in the Z-plane allows the designer to establish specific applications, mainly to confine a signal in a prescribed frequency band as in low-pass, highpass, and band-pass filters; to decompose a signal into two or more sub-bands as in filter-banks, graphic equalizers, sub-band coders, and frequency multiplexers; to modify the frequency spectrum of a signal as in telephone channel equalization and audio graphic equalizers; to model the input-output relationship of a system, such as telecommunication channels, human vocal tract, and music synthesizers [26]. Those features could be directly transferred to circuit theory without loss of generality.

Here, the task of building filter prototypes to select specific frequency components in a signal has been prioritized. We explored the number of zeros (roots of a polynomial filter) of digital filtering structures, placed at $z=-1$. Particularly, we focused on the use of perfect reconstruction filter banks, as those schemes are formed by linear phase analysis and synthesis filters, therefore avoiding the occurrence of undesired disturbances due to phase shifting [26]. 
The design of a perfect reconstruction filter bank could be synthesized in three steps [11, 27]:

i) Selection of a generic polynomial $P_{0}(z)$ that satisfies Eq. (9);

ii) Factorization of the polynomial $P_{0}(z)$ into two polynomials $G_{0}(z)$ and $H_{0}(z)$, where $H_{0}(z)$ contains all zeros only at $z=-1$; and

iii) Determination of impulse responses of low-pass filters $h_{0}[n]$ and $g_{0}[n]$, as well as the ones for highpass filters $h_{1}[n]=(-1)^{n} g_{0}[n]$ and $g_{1}[n]=(-1)^{n+}$ ${ }^{1} h_{0}[n]$, through the "alternating signal" construction [7].

According to digital filter theory [2], the magnitude response $|H(z)|$ of a low-pass digital filter must vanish at the digital frequency $\Omega=\pi \mathrm{rad} /$ sample. In Z-plane, such case corresponds to the condition $z=e^{-i \pi}=-1$, ensuring that the high-frequency components of the input signal are essentially canceled. This condition, $H(-1)=0$, is relevant in wavelet theory $[7,8,16,17]$.

In a filter bank, a root located at $z=-1$, with multiplicity $p$, imposes a major role: the associated wavelet function $\psi_{j}$, ${ }_{k}(x)$ has $p$ vanishing moments, which represents a criterion on how those functions decay toward infinity [8]:

$$
\begin{aligned}
\int_{-\infty}^{\infty} x^{m} \psi_{j, k}(x) d x & =\int_{-\infty}^{\infty} x^{m} 2^{j / 2} \psi_{j, k}\left(2^{j} x-k\right) d x \\
& =0 \quad, m<p,
\end{aligned}
$$

where $j$ and $k$ denote the dilation and translation wavelet factors, respectively. Figure 2 shows the Daubechies wavelets [15], generally used in wavelet filter design, related to vanishing moments.

Although the formulation to design perfect reconstruction wavelet filters is given above, there is no explicit criterion on which design is the best choice. One feature that gives some information on the performance of the designed filters, with respect to their behavior on pass and transition bands, is based on the multiplicity of zeros at $z=-1$, which ensures the orthogonality of the designed wavelets and could be explored [4], being useful to determine the number of vanishing moments of that wavelet family.

Therefore, the $p$ value assumes an important role in wavelet filter design, defining the form of the transition band in filtering: a large $p$ value characterizes low-pass filters with a wider passband and a narrower transition band. In contrast, a wavelet function with a few vanishing moments (a low multiplicity $p$ for roots at $z=-1$ ) produces low-pass filters with a wider transition band and a narrower passband. Exploring those features is convenient to select properly the filter to be used according to the nature of the input signal.

\section{Multiresolution selective filter design}

Over the last few years, the expansion of circuits equations on wavelet basis has been explored [28, 29]. This resulted from the fact that expanding these equations on a wavelet basis gives algebraic systems that can be conveniently solved (by numerical techniques), producing accurate results with a low-computational cost. This

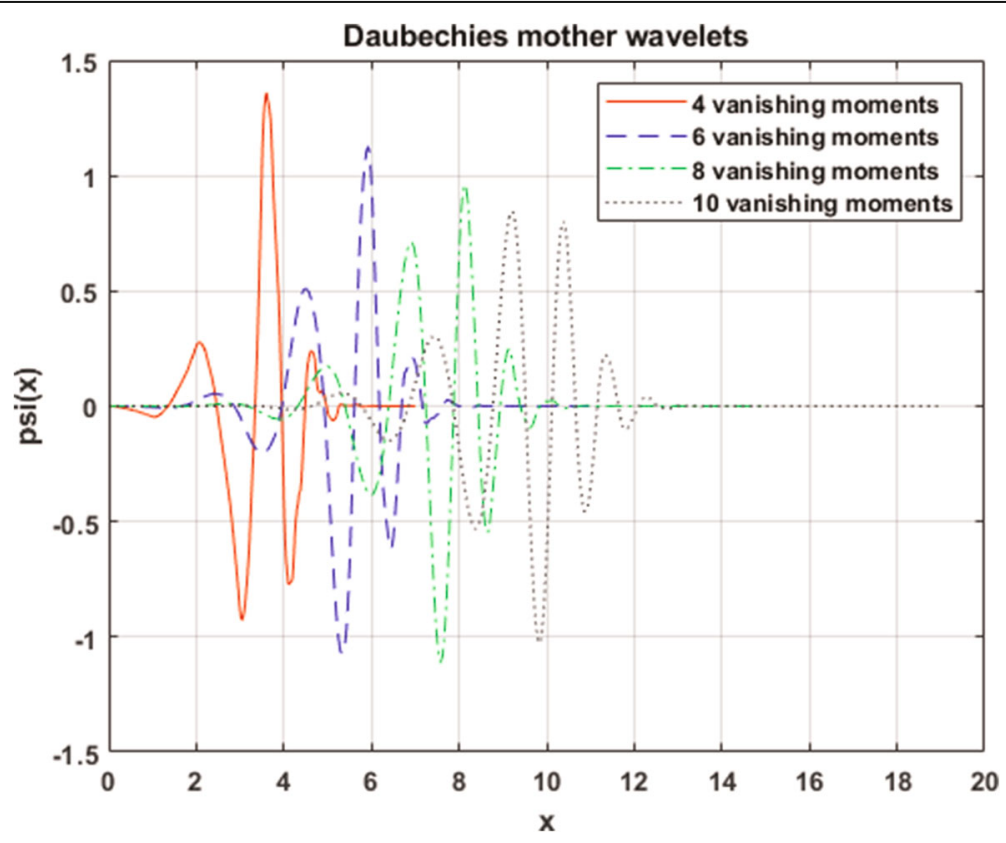

Fig. 2 Daubechies wavelets with different vanishing moments 
feature is particularly evident when irregular signals (such as fast transients) are considered, since the wavelet basis gives a concise representation (i.e., characterized by a reduced number of coefficients). In fact, the Daubechies wavelets, on the interval in which the representation of circuits equations has been found [30], are the most suitable wavelet basis for this type of problems. They are orthonormal wavelet basis, characterized by a certain number of vanishing moments [31], since they offer the best efficiency in terms of accuracy versus calculation time.

Nevertheless, the uneven distribution of signal energy in the frequency domain has made signal decomposition into wavelets an important practical problem. Rate-distortion theory shows that the uneven spectral nature of real-world signals can provide the basis for source compression techniques [32]. The basic concept explored here refers to dividing the signal spectrum into subbands, in a manner that the sub-spectrum with more energy content deserves higher priority for filtering. For example, a slowly varying signal will have predominantly low-frequency components. Therefore, the low-pass subbands contain most of its total energy. If one filters the high-pass analysis sub-bands and reconstructs the signal, it is expected that very little or negligible reconstruction error occurs after this analysis/synthesis operation. The decomposition of the signal spectrum into sub-bands provides the mathematical basis for an important and desirable feature in the signal analysis: the monitoring of signal energy components within the sub-bands is possible, as the sub-band signal can be ranked and processed independently.

Under such considerations and after emphasizing the relevance of roots at $z=-1$ in the previous section, the filter bank design can be analytically developed, specifically through spectral factorization of a polynomial filter $[9,22]$. The construction of a filter bank, according to that method, requires the computation of the roots of a particular product filter

$$
P_{0}^{<D>}(z)=\left(1+z^{-1}\right)^{2(D+1)} Q_{D}(z)
$$

where $Q_{D}(z)$ is a Laurent polynomial with degree $D$ and $2 D$ roots [7].

In order to separate real from complex roots of the polynomial $Q_{D}(z)$, a possible form of $P_{0}^{<D>}(z)$ is here proposed using [30], such that

$$
P_{0}^{<D>}(z)=\left(1+z^{-1}\right)^{2(D+1)} U\left(z^{-1} ; z_{i}\right) V\left(z^{-1} ; r_{j}\right)
$$

where

$$
\begin{aligned}
U\left(z^{-1} ; z_{i}\right)= & \prod_{i=1}^{n^{c q}}\left(1-z_{i} z^{-1}\right)\left(1-z_{i}^{-1} z^{-1}\right)\left(1-\bar{z}_{i} z^{-1}\right) \\
& \times\left(1-\bar{z}_{i}^{-1} z^{-1}\right)
\end{aligned}
$$

and

$$
V\left(z^{-1} ; r_{j}\right)=\prod_{i=1}^{n^{r d}}\left(1-r_{j} z^{-1}\right)\left(1-r_{j}^{-1} z^{-1}\right)
$$

are auxiliary functions obtained from the separation of $P_{0}^{<D>}(z)$ into roots at $z=-1$, complex and real roots, and where $n^{c q}$ and $n^{r d}$ are respectively the numbers of quadruple complex factors and of double real factors in $Q_{D}(z)$. In fact, the precise separation of roots into two sets, the ones located at $z=-1$ and the ones located in other regions in $Z$-plane, is the key element of the proposed algorithm for filter circuits design. It can be shown that $n^{c q}=D / 2$ and $n^{r d}=D \bmod 2$ [33, 34], where 'mod' represents the modulus operator. The Eqs. (16), (17), (18), (19) could be interpreted as

i) For an arbitrary polynomial $F(z)$ with $N$ coefficients, there are $N-1$ roots, from which a subset of $K$ integer roots, $0 \leq K \leq N-1$, may be placed at $z=-$ 1 ;

ii) In spectral factorization, the polynomial of the product filter $P_{0}^{<D>}(z)$ with $N_{P}=4 D+3$ coefficients and $K_{p}=2 D+2$ roots at $z=-1$ is factorized into polynomials; and

iii) Each polynomial corresponds to analysis low-pass filter, $H_{0}(z)$, and synthesis low-pass filter, $G_{0}(z)$, respectively, with $N_{h}$ and $N_{g}$ coefficients and $K_{h}$ and $K_{g}$ roots at $z=-1$.

This factorization leads to the following constraint for the roots at $z=-1$ :

$$
N_{p}=N_{h}+N_{g}-1
$$

and

$$
K_{p}=K_{h}+K_{g} .
$$

The wavelet family, chosen in the design due to its linear phase, suitable for circuit design [30], is the symmetrical orthogonal one, which requires $[8,11]$ :

$$
\begin{aligned}
& N_{h}=K_{h}+4 n_{h}^{c q}+2 n_{h}^{r d}+1, \\
& N_{g}=K_{g}+4 n_{g}^{c q}+2 n_{g}^{r d}+1,
\end{aligned}
$$

and

$$
N_{p}=2 K_{p}-1,
$$

where the subscripts $h$ and $g$ correspond to the analysis and synthesis polynomials, respectively. Non-integer 
values of $n^{c q}$ and $n^{r d}$ correspond to a pair of complex roots and a single real root, respectively. Additionally, $K_{h}$ and $K_{g}$ must be simultaneously even or odd, which imposes

$$
n_{p}^{c q}=n_{h}^{c q}+n_{g}^{c q}
$$

and

$$
n_{p}^{r d}=n_{h}^{r d}+n_{g}^{r d}=1,
$$

where $n_{p}^{c q}$ and $n_{p}^{r d}$ denote the number of pairs of complex and single real roots for both analysis and synthesis stages, respectively. Once defined, the multiplicity of the root at $z=-1$ for low-pass stages of analysis and synthesis steps, Eqs. (22) and (23) could be used to obtain the number of coefficients for each $Q_{D}(z)$ factor. Eqs. (25) and (26) determine the splitting of roots into $H_{0}(z)$ and $G_{0}(z)$ polynomials, whence the low-pass impulse responses $h_{0}[n]$ and $g_{0}[n]$ could be obtained and, simultaneously, $h_{1}[n]$ and $g_{1}[n]$ [35-37], using the alternating signal scheme for perfect reconstruction.

As a result, the designed filtering method for circuits applications consists in partitioning the signal to be filtered into two wavelet sub-bands, with subsequent filtering and wavelet reconstruction stages, described in Fig. 3. The application of wavelet transform splits the signal into two parts: a set of low-frequency components with higher amplitude values, and a set of high-frequency components with lower amplitude values. The portion of the signal energy allocated for each sub-band determines the filtering performance.

\section{Results and discussion}

The formal development of the wavelet filter bank design was presented in Eqs. (16), (17), (18), (19). The next step involves a particular partitioning of the signal energy, described as follows:

i) The multiplicity of roots at $z=-1$ is set for lowpass filters of analysis and synthesis steps. In the simulations, better results, in terms of observing a sharper response in transition band, were obtained for $K_{h}=1$ and $K_{g}=7$, leading to

$$
K_{p}=K_{h}+K_{g}=8
$$

and

$$
D=\frac{K_{p}-2}{2}=3
$$

ii) Under the assignment of the value of $D$, Eqs. (16) and (17) are used to determine the number of coefficients for each factor of $Q_{D}(z)$. Once $D=3$, the number of coefficients for the factors of $Q_{3}(z)$ must be assigned, since $N_{P}=4 D+3=15$ and $K_{p}=$ $2 D+2=8$. Defining $n_{h}^{c q}=0$ and $n_{h}^{r d}=0$, it is obtained:

$$
N_{h}=K_{h}+1=2 .
$$

Similarly, defining $n_{g}^{c q}=1$ and $n_{g}^{r d}=1$, it can be shown that

$$
N_{g}=K_{g}+4+2+1=14 .
$$

iii) Choosing a single root at $z=-1$ for the low-pass filter into analysis step, $H_{0}(z)$, the low-pass impulse responses $h_{0}[n]$ and $g_{0}[n]$ are obtained, and hence $h_{1}[n]$ and $g_{1}[n]$, through the "alternating signal" scheme.

The resulting low-pass filters for analysis and synthesis steps in the $\mathrm{Z}$ domain, according to the steps performed in (i)-(iii) and developed from Eqs. (16), (17), (18), and (19), are respectively obtained as

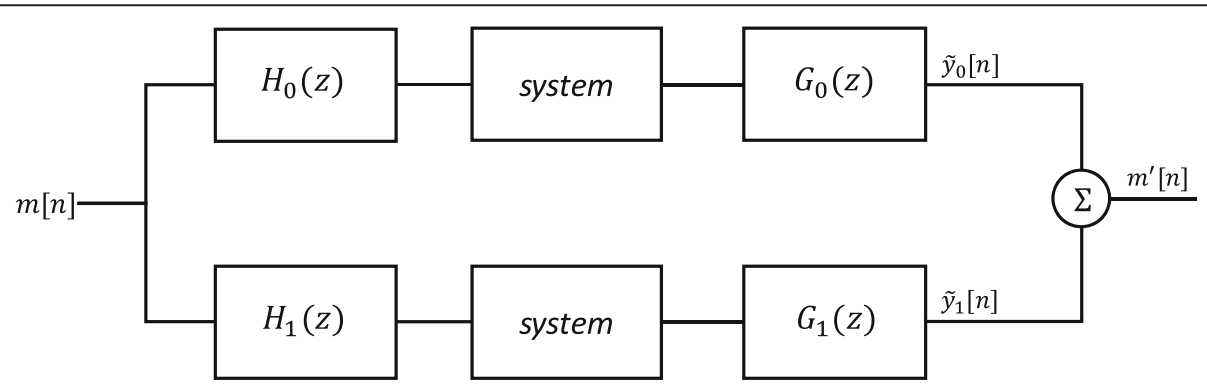

Fig. 3 Block diagram for a reconstruction two-channel filter bank 


$$
H_{0}(z)=\frac{\sqrt{2}}{2} z^{-6}+\frac{\sqrt{2}}{2} z^{-7}=\frac{\sqrt{2}}{2} z^{-6}\left(1+z^{-1}\right)
$$

and

$$
\begin{aligned}
G_{0}(z) & =-0,003452\left(1-z^{-1}-8,8 z^{-2}+8,8 z^{-3}+40,2 z^{-4}\right. \\
& -40,2 z^{-5}-204,8 z^{-6}-204,8 z^{-7}-40,2 z^{-8} \\
& \left.+40,2 z^{-9}+8,8 z^{-10}-8,8 z^{-11}-z^{-12}+z^{-13}\right) .
\end{aligned}
$$

Once defined, the responses $H_{0}(z)$ and $G_{0}(z)$, a wavelet family is designed for use in a filter bank, as both filters form the proposed filter bank in a condensed manner. Figure 4 shows the scaling function $\phi(x)$ and wavelet function $\psi(x)$, corresponding to the designed filter bank, compared to the simplest wavelet functions, the Haar functions [17], which also have a single root at $z=-1$ in its analysis low-pass stage, being therefore adequate for comparison due to its simplicity of representation. Figure 5 shows the zero map for the designed low-pass filters. Those simulations were performed using $\mathrm{MATLAB}^{\circ}$ software.

The designed synthesis low-pass or high-pass filters could be used in circuit theory, since their roots are known. This is a noticeable advantage in comparison to other filtering methods, based uniquely on the frequency response modeling. Within this method, both filter response and location of roots are performed, allowing a more flexible circuit synthesis. The frequency response for the synthesis low-pass stage is shown in Fig. 6 (normalized for unitary gain at $\Omega=0 \mathrm{rad} / \mathrm{sample}$ ), in comparison to a second-order continuous-time low-pass filter with cutoff frequency of $1 \mathrm{kHz}$, converted to its digital form by bilinear transformation, and, for instance, to a windowed FIR filter-a typical filter in circuit synthesis [36].

Figure 7 shows the frequency response in decibel for each filter tested in Fig. 6, when compared to an ideal digital low-pass filter. These results indicate that the proposed algorithm presents better performance than other filtering methods: at $\Omega=\pi / 2 \mathrm{rad}$, the frequency response of the proposed filter shows a stable behavior with lower attenuation, while presenting a fast convergence to zero at higher frequencies $(\Omega=\pi \mathrm{rad})$. Several sets of zeros were tested to factorize the product filter, being selected the one with the best signal energy balance, using a few filter coefficients.

Figure 8 shows that the proposed wavelet filter bank ensures high stop-band attenuation, despite its simpler implementations when compared to typical filter design methods [38, 39]. Finally, the filter developed here is compared to other wavelet filters, in order to validate the design method. Figure 8 shows the frequency response of the designed synthesis lowpass filter, when compared to others obtained from alternative wavelet functions, such as the Haar and 'orthogonal db8' (Daubechies) ones [11, 12]. The

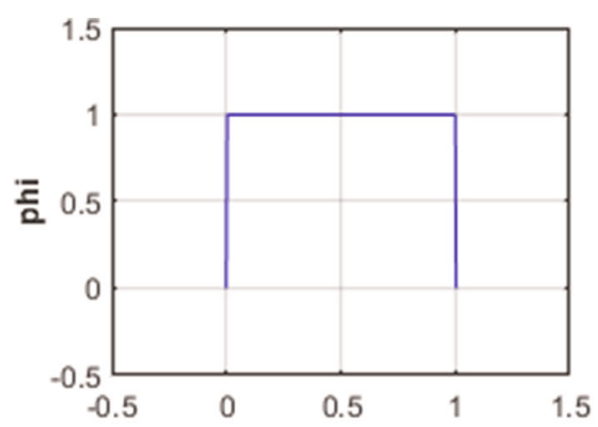

(a)

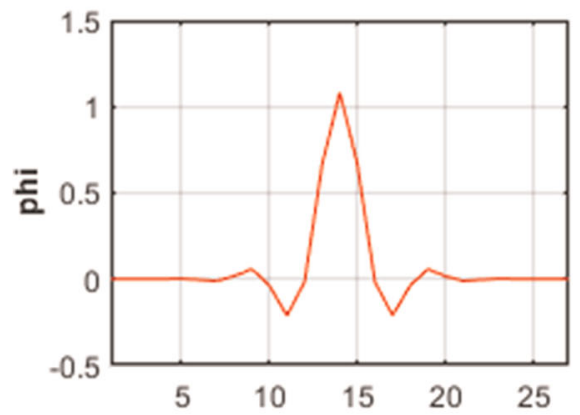

(c)

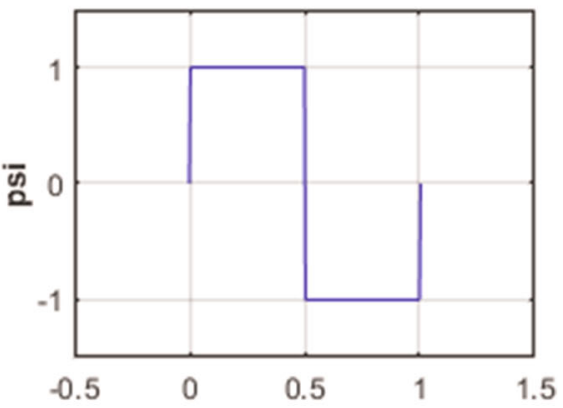

(b)

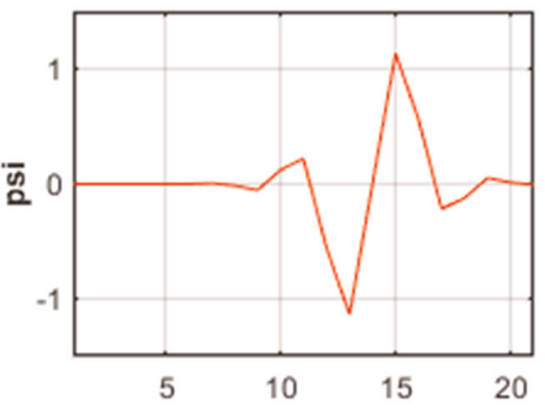

(d)

Fig. 4 a Haar scaling function. b Haar mother wavelet. c Designed scaling function. d Designed mother wavelet 


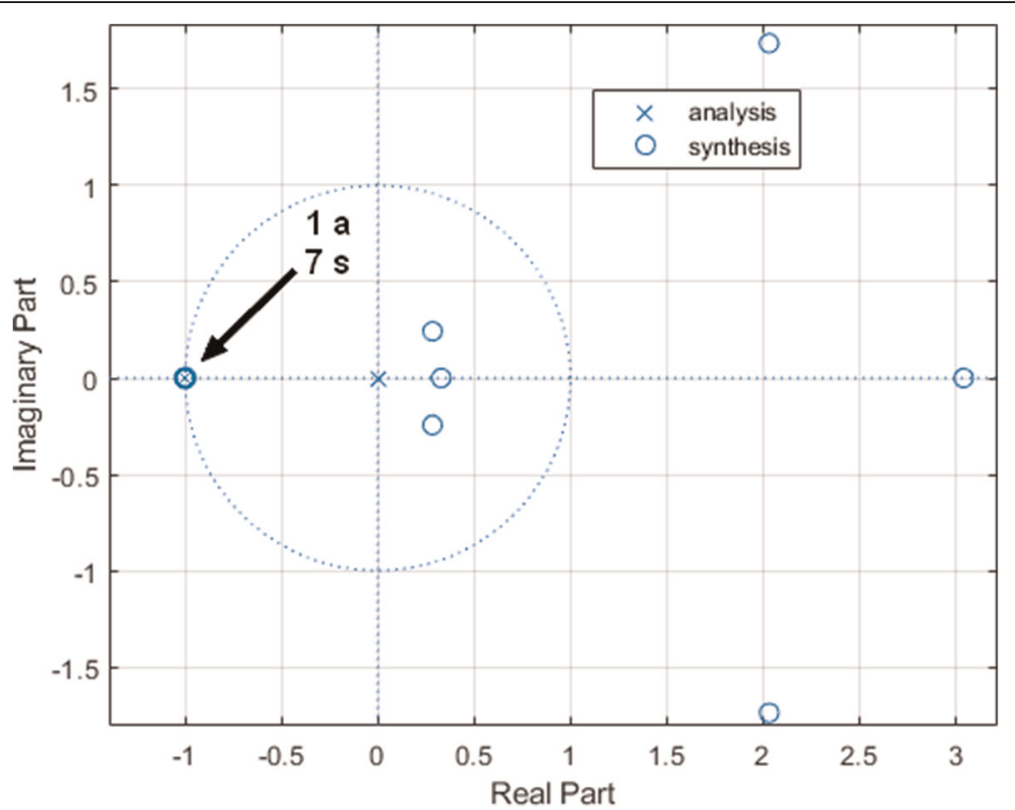

Fig. 5 Roots of designed low-pass filters in analysis and synthesis steps. The multiplicity of roots at $z=-1$ for each stage ( 1 for analysis and 7 for synthesis) is pointed out in the figure

proposed algorithm is well suited to behave as a good window function in several signal processing applications, showing a higher to accurately resolve noise and distortion components in the frequency spectrum, emphasizing the performance of the proposed model as that is measured by the behavior of the frequency response of the whole filter bank.

The present strategy shows that the concept of wavelets can be explored in the context of circuit theory applications and becomes an additional model to be compared to other well-established filters [40-46], as it can bring out the orthogonal and the multiresolution properties of the wavelet, improving the efficiency of the calculations. As minor disadvantages, this technique requires a pre-processing of the signals to be filtered in the wavelet domain, such as symmetric extension (a mandatory procedure in wavelet filter design [47-52]).

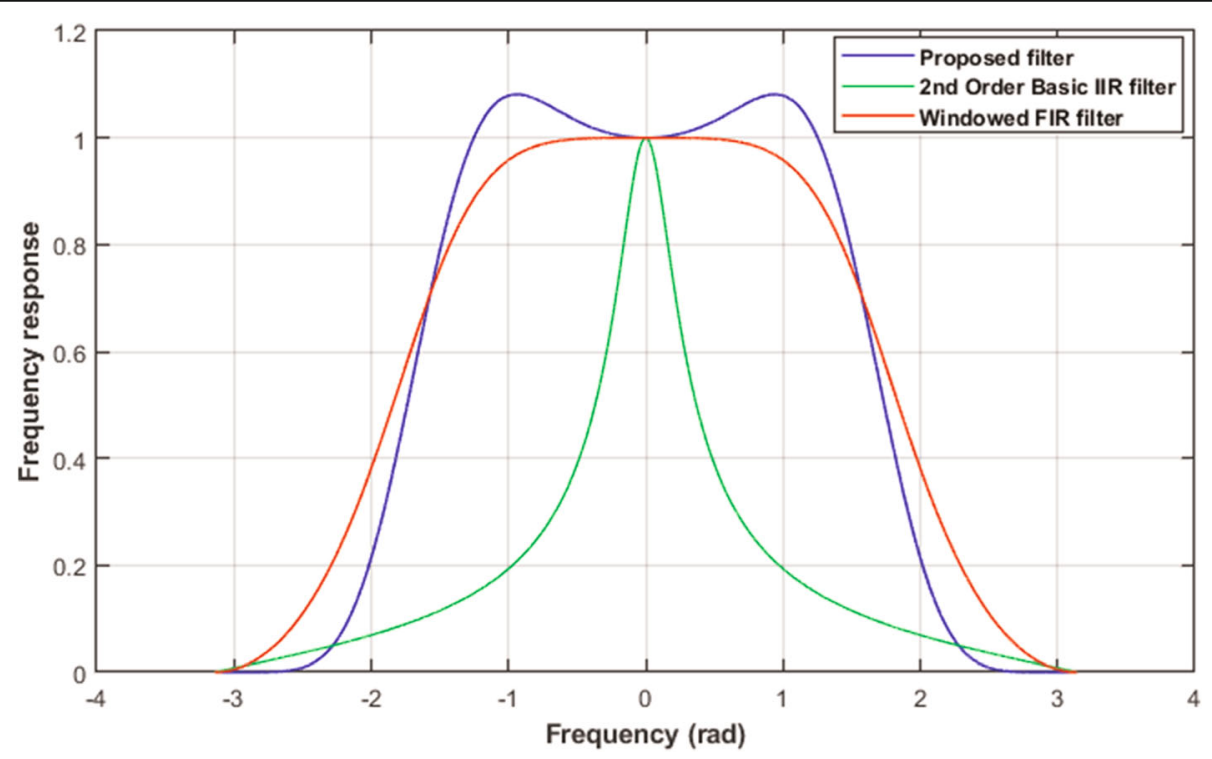

Fig. 6 Frequency response for the proposed low-pass filter, compared to a continuous-time low-pass filter after digitization 


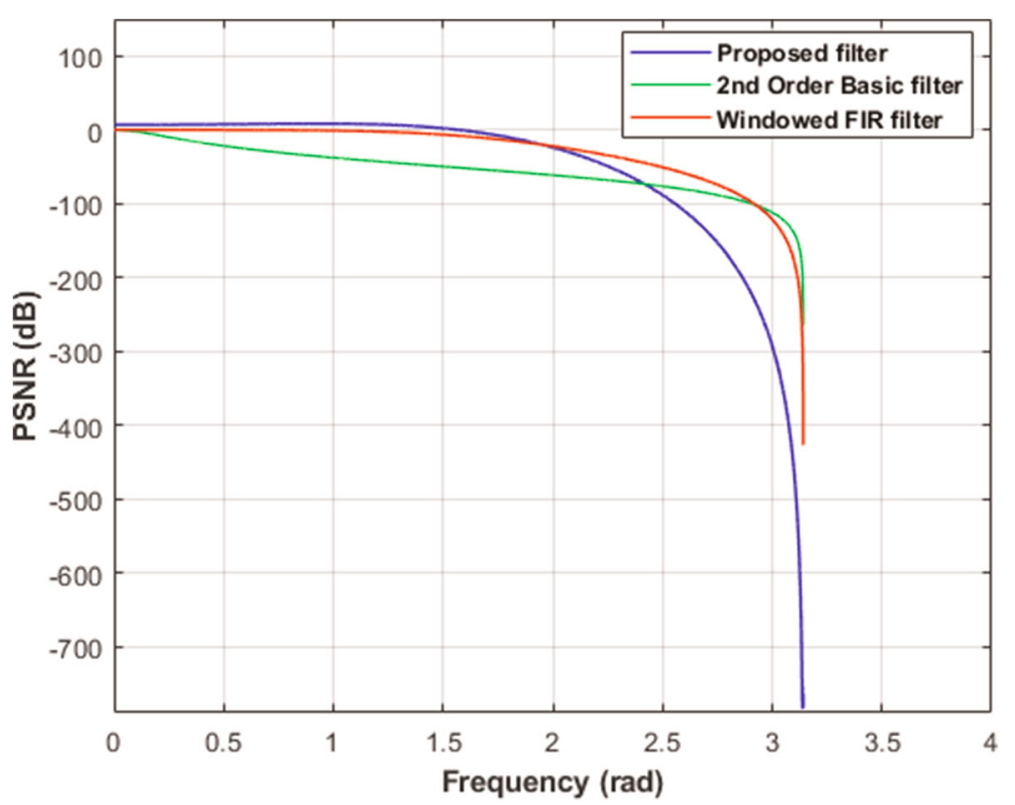

Fig. 7 Frequency response absolute values (in decibels) for the proposed filter design, compared to a digitized continuous-time low-pass filter. The relative amplitude is varied from zero to unity

\section{Conclusions}

In summary, the use of wavelet filter banks in circuit theory allows the implementation of simple filter stages, in terms of the practical formulation involved in that task. The separation of signals into low- and high-frequency values is noticeably efficient, as the energy of the signals involved could be properly partitioned. Nevertheless, the implementation of spectral factorization to wavelet filter design is adequate to build fast and simple filter circuits, in the sense that the design approach based on the multiplicity of roots at $z=-1$ is shown to be elegant and efficient, combining mathematical consistency and good performance. Additionally, the good approximation obtained by wavelets for high-frequency components encourages the proposition of filter schemes in power electronics circuits, where drastic switching procedures are observed. Nevertheless, this filter design method is significantly effective in obtaining a fast transition out of the passband in the frequency domain, as well as the numerical efficiency of wavelets

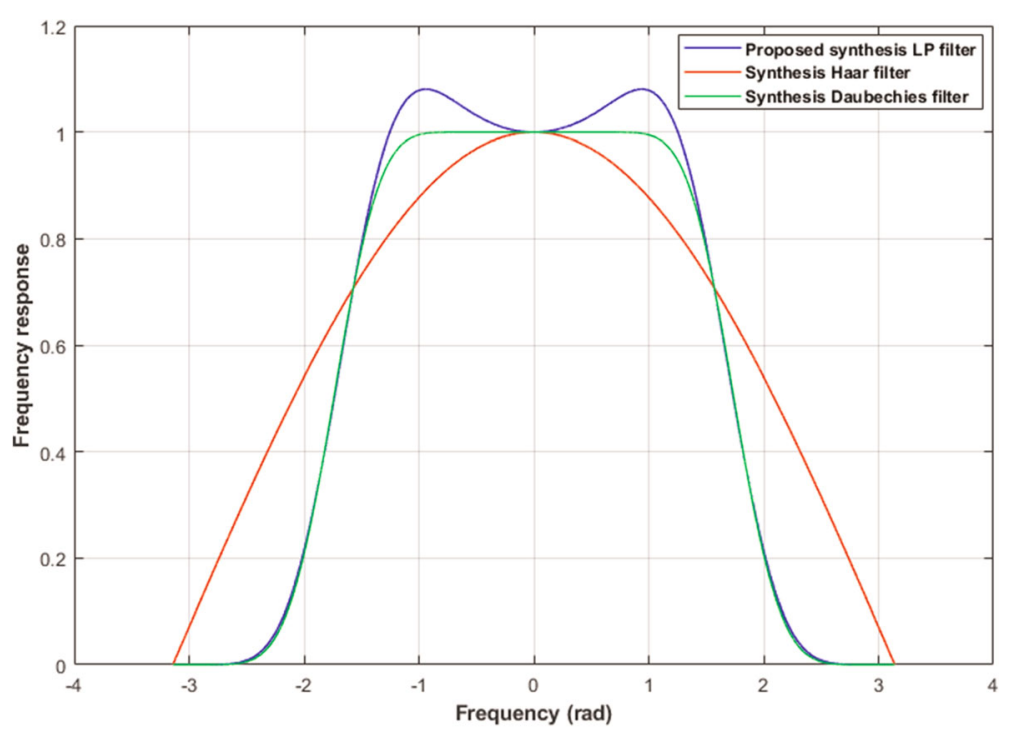

Fig. 8 Frequency response of synthesis low-pass filters for different mother wavelet functions 
makes the method effective for fast and reliable numerical circuit simulations, despite its mathematical simplicity. The use of wavelets is also preferable in comparison to other filtering schemes, due to the easy time-frequency duality [48]. This shows the relevant contribution of the proposed filter, as other windowing functions do not offer enough side-lobe attenuation to be used in filtering applications. As a future development, optimization approaches could be considered for comparison, since they are not included in such analysis due to the fact they produce high computational effort for implementation, like many other IIR filter schemes.

\section{Abbreviations}

FIR: Finite impulse response; IIR: Infinite impulse response; PSNR: Peak signalto-noise ratio

\section{Acknowledgements}

Not applicable.

\section{Authors' contributions}

SRMP developed the methodology and carried the simulations. MLN and JFJ prepared the manuscript. All authors read and approved the final manuscript.

\section{Funding}

The authors acknowledge partial funding from Brazilian agencies CAPES (Coordenação de Aperfeiçoamento de Pessoal de Nível Superior) and CNPq (Conselho Nacional de Desenvolvimento Científico e Tecnológico).

\section{Availability of data and materials}

All data were generated by the equations presented in the methods sections, which are presented in the respective figures. Data sharing is not applicable to this article.

\section{Consent for publication}

Not applicable.

\section{Competing interests}

The authors declare that they have no competing interests.

Received: 14 January 2019 Accepted: 8 July 2019

Published online: 22 July 2019

\section{References}

1. A. Ambede, K.G. Smitha, A.P. Vinod, Flexible low complexity uniform and nonuniform digital filter banks with high frequency resolution for multistandard radios. IEEE Trans. Very Large Scale Integration (VLSI) Syst. 23(4), 631-641 (2015)

2. Z. Fang, S. Penglang, Construction of nonuniform DFT modulated filter banks via phase modulation. J. Electron. Inf. Technol. 39(3), 2169-2174 (2017)

3. J.Z. Jiang, F. Zhou, P.L. Shui, Lifting-based design of two-channel biorthogonal graph filter bank. IET Signal Process. 10(6), 670-675 (2016)

4. J.Z. Jiang, F. Zhou, P.L. Shui, S. Ouyang, Theory and design of twodimensional DFT modulated filter bank with arbitrary modulation and decimation matrices. Digit. Signal Process. 44, 123-130 (2015)

5. D.B.H. Tay, Z.P. Lin, Design of near orthogonal graph filter banks. IEEE Signal Process. Lett. 22(6), $701-704$ (2015)

6. F. Zhou, P.L. Shui, J.Z. Jiang, Design of two-dimensional modified DFT modulated filter banks based on Lagrange multiplier method. J. Electron. Inf. Technol. 39(5), 1261-1265 (2017)

7. Y.S. Won, K.U. Bae, N.H. Myung, Design method for bandpass filter with enhanced stopband rejection using spiral SIRs. Electron. Lett. 48(17), 10671068 (2012)

8. I. Daubechies, Orthonormal bases of compactly supported wavelets. Commun. Pure Appl. Math. 41, 909-996 (1988)

9. J. Shen, G. Strang, The zeros of the Daubechies polynomials. Proc. Am. Math. Soc. 124(12), 3819-3833 (1996)
10. C. Vonesch, T. Blu, M. Unser, Generalized Daubechies wavelet families. IEEE Trans. Signal Process. 55(9), 4415-4429 (2007)

11. G. Strang, Creating and comparing wavelets (Technical Paper, Department of Mathematics, Massachusetts Institute of Technology, Cambridge, 1994), pp. 1-10

12. C. Taswell, Constraint-selected and search-optimized families of Daubechies wavelet filters computable by spectral factorization (Technical Paper, Computational Toolsmiths, Stanford, 2000)

13. M. Oishi, S. Moro, T. Matsumoto, A modified method for circuit analysis using Haar wavelet transform with adaptive resolution - For circuits with waveform with sharp convex ranges. European Conf. Circuit Theory Design (ECCTD) 1(1), 299-302 (2009)

14. M. Oishi, S. Moro, T. Matsumoto, Accuracy of circuit analysis method using wavelet transform with adaptive resolutions. IEEE Region 10 Conference (TENCON) 1(1), 1177-1181 (2010)

15. X.M. Xie, S.C. Chan, T.I. Yuk, Design of perfect-reconstruction nonuniform recombination filter banks with flexible rational sampling factors. IEEE Trans. Circuits Syst. I - Regular Papers 52(9), 1965-1981 (2005)

16. L. Fang, W. Zhong, Q. Zhang, Design of M-channel linear-phase nonuniform filter banks with arbitrary rational sampling factors. IET Signal Process. 10(2), 106-114 (2016)

17. W. Zhang, Y. Chen, Z. Dou, J. Hu, Low complexity and narrow transition band filter banks for software defined radio applications (IEEE 13th International Conference on Signal Processing (ICSP), Chengdu, 2016), pp. 1171-1175

18. B. Boashash (ed.), Time-frequency signal analysis and processing-a comprehensive reference, 2nd edn. (Academic Press, New York, 2015)

19. P.P. Vaidyanathan, Filter banks in digital communications. IEEE Circuits Syst. Mag. 1(2), 4-25 (2001)

20. X. Chen, F.J. Harris, E. Venosa, B.D. Rao, Non-maximally decimated analysis/ synthesis filter banks: Applications in wideband digital filtering. IEEE Trans. Signal Process. 62(4), 852-867 (2014)

21. S.G. Mallat, A theory for multiresolution signal decomposition: the wavelet representation. IEEE Trans. Pattern Anal. Mach. Intellig. 11(7), 674-693 (1989)

22. I. Daubechies, Ten lectures on wavelets, CBMS-NSF Regional Conf. Series in Appl. Mathematics (SIAM) 61, Philadelphia, PA, 1992

23. A. Salazar et al., Automatic credit card fraud detection based on non-linear signal processing. IEEE Int. Carnahan Conf. Security Technol. (ICCST) 1(1), 207-212 (2012)

24. K. Pourvoyeur, R. Heidger, Secure ADS-B usage in ATC tracking. Tyrrhenian Int. Workshop Digit. Commun. Enhanced Surveill. Aircraft Vehicles (TIWDC/ ESAV) 1(1), 35-40 (2014)

25. R. Parseh, K. Kansanen, Diversity effects in the estimation of a gauss-Markov process over a fading channel. IEEE Int. Conf. Commun. (ICC) 1(1), 46444649 (2014)

26. K. Sung-Eun et al., Sound transmission through the human body with digital weaver modulation (DWM) method. Ann. IEEE Syst. Conference (SysCon) 1(1), 176-179 (2014)

27. M. Vetterli, C. Herley, Wavelets and filter banks: Theory and design. IEEE Trans. Signal Process. 40(9), 2207-2232 (1992)

28. E.S. Filatova, D.M. Filatov, A.D. Stotckaia, G. Dubrovskiy, Time series dynamics representation model of power consumption in electric load forecasting system. IEEE NW Russia Young Researchers Electrical Electron. Eng. Conference (ElConRusNW) 1(1), 175-179 (2015)

29. S.N. Mate, Identification of time-varying system with wavelet based approach using multiple wavelet basis functions. 2nd Int. Conference Electron. Commun. Syst. (ICECS) 1(1), 441-446 (2015)

30. N.W.A. Lidula, R.M.M.P. Nishshanka, S.B. Wijesundara, W.L.K.D. Wijemanna, W. N.L. Weerakkody, Designing a microgrid test system for transient analysis. Moratuwa Eng. Res. Conference (MERCon) 1(1), 88-93 (2015)

31. A. Cohen, I. Daubechies, B. Jawerth, P. Vial, Multiresolution analysis wavelets and fast algorithms on the interval. C. R. Acad. Sci. Paris, ser. i Math 316 , 417-421 (1992)

32. A.N. Akansu, R.A. Haddad, Multiresolution Signal Decomposition - Transforms, Subbands and Wavelets, 2nd edn. (Academic Press, 2001)

33. H. Youngmi, P. Hyungju, Z. Fang, Multi-D wavelet filter bank design using Quillen-Suslin theorem for Laurent polynomials. IEEE Trans. Signal Process. 62(20), 5348-5358 (2014)

34. A.I. Markushevich, Theory of functions of a complex variable, 2nd edn. (AMS Chelsea Publishing, New York, 2011)

35. J.D. Certic, Efficient digital filter structures based on allpass filter sections, Telecommunications Forum (TELFOR), 19th edn. (2011), pp. 743-750 
36. L. Jian-ao, Multidimensional PR filter banks with FIR filters. Int. Conf. Commun. Circuits Syst. Proc. 1, 162-166 (2006)

37. K. Avci and E. Gümüşsoy, Design of exponential window based M-channel cosine modulated filter banks, 24th Signal Processing and Communication Application Conference (SIU), Zonguldak 845-848, 2016

38. S. Dhabu, V.A. Prasad, Design of modified second-order frequency transformations based variable digital filters with large cutoff frequency range and improved transition band characteristics. IEEE Trans. Very Large Scale Integration (VLSI) Syst. 24(2), 413-420 (2016)

39. Z. Songbai, Z. Lei, General synthesis method for symmetrical even-order Chebyshev bandpass filter. Microwave Conf. Proc. (APMC) 1(1), 667-669 (2012)

40. R. Schaumann, H. Xiao, M.E. Van Valkenburg, Design of analog filters, 2nd edn. (Oxford University Press, USA, 2009)

41. D. Wei, A.V. Oppenheim, Linear programming algorithms for sparse filter design. IEEE Trans. Signal Process. 58(3), 1605-1617 (2010)

42. D. Wei, A.V. Oppenheim, A branch-and-bound algorithm for quadraticallyconstrained sparse filter design. IEEE Trans. Signal Process. 61(4), 1006-1018 (2013)

43. S. Preethi, M.J. Sheela, Area efficient high and low pass filter design for DWT applications. Int. Conf. Electron. Commun. Syst. (ICECS) 1, 1-5 (2014)

44. F. Ramirez-Echeverria, A. Sarr, Y.S. Shmaliy, Optimal memory for discretetime FIR filters in state-space. IEEE Trans. Signal Process. 62, 557-561 (2014)

45. F. Ding, Y. Wang, J. Ding, Recursive least squares parameter identification algorithms for systems with colored noise using the filtering technique and the auxiliary model. IEEE Trans. Digital Signal Process. 37, 100-108 (2015)

46. Y. Liu, F. Ding, Y. Shi, An efficient hierarchical identification method for general dual-rate sampled-data systems. Automatica 50, 962-970 (2014)

47. A. Aggarwal, M. Kumar, T.K. Rawat, et al., Optimal design of 2D FIR filters with quadrantally symmetric properties using fractional derivative constraints. Circuits Syst. Signal Process. 35(6), 2213-2257 (2016)

48. N. Rashmi, G. Begum, V. Singh, ECG denoising using wavelet transform and filters (International Conference on Wireless Communications, Signal Processing and Networking (WiSPNET), Chennai, 2017), pp. 2395-2400

49. M. Sharma, P.V. Achuth, R.B. Pachori, V.M. Gadre, A parametrization technique to design joint time-frequency optimized discrete-time biorthogonal wavelet bases. Signal Process., 107-120 (2017)

50. X. Zhang, Design of two channel biorthogonal graph wavelet filter banks with half-B and kernels. IEICE Trans. Fundamentals Electron. Commun. Comp. Sci., 1743-1750 (2017)

51. J. Yli-Kaakinen, M. Renfors, Optimized reconfigurable fast convolution-based transmultiplexers for flexible radio access. IEEE Trans. Circuits Syst. II Express Briefs 65, 130-134 (2018)

52. J. Datta, H.P. Lin, Fast convolution filter-bank based non-orthogonal multiplexed cognitive radio (NOMCR) receiver design using cyclostationarity based FRESH filtering. Sensors 18(6), 1930 (2018)

\section{Publisher's Note}

Springer Nature remains neutral with regard to jurisdictional claims in published maps and institutional affiliations.

\section{Submit your manuscript to a SpringerOpen ${ }^{\circ}$ journal and benefit from:}

- Convenient online submission

- Rigorous peer review

- Open access: articles freely available online

- High visibility within the field

- Retaining the copyright to your article

Submit your next manuscript at $\boldsymbol{\nabla}$ springeropen.com 